\title{
SMA ist behandelbar geworden
}

Fragestellung: Kann die spinale Muskelatrophie (SMA) durch den Apoptoseinhibitor Olesoxim in ihrem Verlauf beeinflusst werden?

Hintergrund: Die SMA ist eine neuromuskuläre Erkrankung, die aufgrund eines Gendefekts zu verminderter Produktion von SMN-Protein und zu einem Funktionsverlust der Motoneurone mit klinisch schwerwiegender Muskelschwäche und Atrophie führt.

Seit diesem Jahr ist mit Nusinersen, einem Antisense-Oligonukleotid, erstmals ein Medikament zur Therapie der SMA zugelassen. Die Gabe von Nusinersen erfolgt intrathekal und der primäre Wirkmechanismus beruht auf dem Prinzip, durch Veränderungen des SplicingProzesses die Menge an

Bertini E, Dessaud E, Mercuri E et al. Safety and efficacy of olesoxime in patients with type 2 or non-ambulatory type 3 spinal muscular atrophy: a randomised, double-blind, placebocontrolled phase 2 trial. Lancet Neurol 2017; 16: 513-22 SMN-Protein $\mathrm{zu}$ erhöhen. Möglicherweise sind jedoch für den Funktionsverlust der Motoneurone weitere pathologische mitochondriale Prozesse verantwortlich, die durch den Wirkstoff Olesoxim moduliert werden.
Patienten und Methodik: In eine randomisierte doppelblinde placebokontrollierte Phase-2-Studie wurden insgesamt 165 Patienten im Alter von drei bis 25 Jahren mit SMA-Typ-2 und -3 eingeschlossen. Der primäre Zielparameter nach Gabe von Olesoxim oder Placebo über 24 Monate war die Funktionsverbesserung gemessen anhand einer etablierten Motorskala (MFM).

Ergebnisse: Die MFM-Summenwerte zeigten nach 24 Monaten einen positiven Trend zugunsten von Olesoxim (0,18 Punkte) im Vergleich zu Placebo (- 1,82 Punkte), der jedoch statistisch nicht signifikant war. Es zeigte sich jedoch eine im Vergleich zu Placebo signifikante Verbesserung von MFM-Werten nach sechs und nach 18 Monaten nach Einnahme von Olesoxim. Zudem war die Anwendung von Olesoxim sicher und die Anzahl unerwünschter Arzneimittelwirkungen unterschied sich nicht im Placebovergleich.

Schlussfolgerungen: Mit Olesoxim steht zukünftig möglicherweise ein Medikament zur Verfügung, das motorische Funktionen über einen Zeitraum von 24 Monaten aufrechterhält und komplementär zu Antisense-Oligonukleotiden die Therapie der SMA verbessern könnte.

\section{- Kommentar von Claudia Wurster und Albert C. Ludolph, Ulm}

\section{Komplementärer Ansatz zu Antisense-Nukleotiden erscheint plausibel}

Die spinale Muskelatrophie (SMA) ist eine behandelbare Erkrankung! Diese Aussage wäre noch vor fünf Jahren unglaubwürdig gewesen. Natürlich war die Entwicklung der Antisense-Oligonukleotid-Therapie der entscheidende Meilenstein in der Behandlung der SMA, doch die positiven Hinweise auf eine Wirksamkeit von Olesoxim bei guter Verträglichkeit stärken die Eingangsaussage deutlich. Anhand der Ergebnisse kann man davon ausgehen, dass Olesoxim die Progredienz der SMA verlangsamt, ein weiterer Vorteil der Substanz liegt in seiner oralen Verabreichungsform. Hervorzuheben ist jedoch, dass mit Olesoxim ein pharmakologischer Wirkmechanismus verfolgt wurde, der nicht die Erhöhung der SMN-Proteinkonzentration zum Ziel hat, sondern eine für die SMA relevante mitochondriale Dysfunktion moduliert. Ein komplementärer Einsatz von Olesoxim zu Antisense-Oligonukleotiden wie Nusinersen erscheint damit plausibel.

\section{Multipotente adulte Vorläuferzellen beim ischämischen Schlaganfall}

Fragestellung: In einer randomisierten, doppelblinden, placebokontrollierten Phase-II-Studie wurden bei Patienten mit akutem ischämischen Schlaganfall die Sicherheit und die Wirksamkeit von multipotenten adulten mesenchymalen Vorläuferzellen untersucht.
Hintergrund: Tierexperimentelle Studien wiesen inzwischen überzeugend nach, dass die intravenöse Transplantation allogener adulter, mesenchymaler Vorläuferzellen beim ischämischen Schlaganfall günstige Effekte auf neurologische Funktionserholung, Gewebereorganisation und neuronale Plastizität hat. Nicht 